\title{
Markedly different genome arrangements between serotype a strains and serotypes b or $c$ strains of Aggregatibacter actinomycetemcomitans
}

\author{
Weerayuth Kittichotirat', Roger Bumgarner ${ }^{1}$, Casey Chen $^{2 *}$
}

\begin{abstract}
Background: Bacterial phenotype may be profoundly affected by the physical arrangement of their genes in the genome. The Gram-negative species Aggregatibacter actinomycetemcomitans is a major etiologic agent of human periodontitis. Individual clonal types of A. actinomycetemcomitans may exhibit variable virulence and different patterns of disease association. This study examined the genome arrangement of A. actinomycetemcomitans using the genome sequences of serotypes a-c strains. The genome alignment and rearrangement were analyzed by the MAUVE and the GRIMM algorithms. The distribution patterns of genes along the leading/lagging strands were investigated. The occurrence and the location of repeat sequences relative to the genome rearrangement breakpoints were also determined.

Results: The genome arrangement of the serotype a strain D7S-1 is markedly different from the serotype b strain HK1651 or the serotype c strain D11S-1. Specific genome arrangements appear to be conserved among strains of the same serotypes. The reversal distance between D7S-1 and HK1651 by GRIMM analysis is also higher than the within-species comparisons of 7 randomly selected bacterial species. The locations of the orthologous genes are largely preserved between HK1651 and D11S-1 but not between D7S-1 and HK1651 (or D11S-1), irrespective of whether the genes are categorized as essential/nonessential or highly/nonhighly expressed. However, genome rearrangement did not disrupt the operons of the A. actinomycetemcomitans strains. A higher proportion of the genome in strain D7S-1 is occupied by repeat sequences than in strains HK1651 or D11S-1.

Conclusion: The results suggest a significant evolutionary divergence between serotype a strains and serotypes $\mathrm{b} / \mathrm{c}$ strains of $\mathrm{A}$. actinomycetemcomitans. The distinct patterns of genome arrangement may suggest phenotypic differences between serotype $a$ and serotypes b/c strains.
\end{abstract}

\section{Background}

Bacterial genomes are relatively plastic and may display significant variation even among strains within the same species. The variation is often due to large scale genome deletion and/or gene acquisition by horizontal gene transfer of elements such as genomic islands [1]. Consequently, genome content can be divided into a core gene pool and a flexible gene pool [1-3]. The variation in genome content is thought to be a key factor in the evolution of bacterial pathogens. Moreover, the variation

\footnotetext{
* Correspondence: ccchen@usc.edu

${ }^{2}$ Division of Periodontology, Diagnostic Sciences and Dental Hygiene, Herman Ostrow School of Dentistry of the University of Southern California, Los Angeles, CA, USA

Full list of author information is available at the end of the article

in genome arrangement (ie, the physical arrangement of genes) may also affect the virulence of the bacteria.

Genome rearrangement may occur via illegitimate recombination and homologous recombination among repeated elements and duplicated genes such as rDNA operons, and may also occur after horizontal gene transfer or phage infection. While genome rearrangements occurred frequently in laboratory cultures of Escherichia coli, very few were fixed since the divergence of $E$. coli and Salmonella enterica $100 \mathrm{MYA}[4,5]$. Most of the rearrangements presumably have adverse effects on the bacteria due to the constraints placed by cellular processes such as replication, transcription and gene regulation [6,7]. Consequently, the genome rearrangements between closely related bacteria commonly involve 
large-scale inversions along the axis of the origin (Ori) and the terminus (Ter) of replication [8-10]. Such changes presumably have much less deleterious effects due to preservation of the gene locations relative to replication and other cellular processes.

Gram-negative facultative Aggregatibacter actinomycetemcomitans is a member of the Pasteurellaceae family [11]. It is a recognized pathogen in periodontitis and extra-oral infections. There are 6 distinct serotypes; each serotype may represent a distinct clonal lineage of A. actinomycetemcomitans. Depending on the disease status and race/ethnicity of the subjects dominant serotypes within the study populations may include serotypes a, b, c, and e [12,13]. Serotypes $d$ and $f$ are in general detected less frequently $[12,13]$.

Certain clonal lineages of $A$. actinomycetemcomitans, such as the JP-2 clone, appear to exhibit a high degree of virulence [14-20]. However, other non-JP2 A. actinomycetemcomitans strains were also associated with aggressive periodontitis and are presumed to be highly virulent as well $[13,21]$. Interestingly, in the study of a subgingival microbial community by Socransky et al, A. actinomycetemcomitans serotype a strains were a component of the green complex, while A. actinomycetemcomitans serotype b strains were not in association with other bacterial species [22]. It seems plausible that A. actinomycetemcomitans strains are distinct in their phenotypes, pathogenic mechanisms, and functional roles in the subgingival microbial communities, which may result in different patterns of disease association.

To understand the molecular basis of the variations of virulence in $A$. actinomycetemcomitans, we sequenced and compared the genome content and structure of A. actinomycetemcomitans strains recovered from different clinical settings. We have obtained initial evidence for significant genome content variations among strains $[23,24]$. This study further examined the differences in the genome arrangement among $A$. actinomycetemcomitans strains of serotypes a-c. The results showed striking differences in the genome arrangements of serotype a strains compared to serotypes b or c strains. Such differences indicate divergent evolutionary pathways and possibly phenotypic differences between serotype a and serotype b/c strains of $A$. actinomycetemcomitans.

\section{Results}

\section{Genome rearrangement between $A$.} actinomycetemcomitans strains

The results of genome comparison by MAUVE for $A$. actinomycetemcomitans are shown in Figure 1. The reversal distances obtained by GRIMM (for A. actinomycetemcomitans and other bacterial species) are summarized in Table 1. For comparison between D11S-1 and HK1651 there are 9 locally collinear blocks (LCBs) with a minimum weight of 8,386 identified by the progressive MAUVE (Figure 1a), with a reversal distance of 5 (Figure 1b). The reversal distance of 5 can be viewed as a hypothetical 5-step inversion process to convert the genome arrangement of one strain to the other strain. The rearrangements involved at least one large scale genomic inversion along the axis of Ori-Ter (see later section for more explanation). For the comparison between D7S-1 and HK1651 there are 102 LCBs with a minimum weight of 35 (Figure 1c) and a reversal distance of 80, which was not only greater than that between HK1651 and D11S-1 but also greater than those between strains in other bacterial species (Table 1).

Seventy genome breakpoints of D7S-1 (in comparison to HK1651) were randomly selected for PCR analysis. All examined sites yielded PCR products of the expected sizes (see Additional File-1). Sixteen of the 70 PCR products were also sequenced and the results confirmed the sequences expected of the breakpoint regions (see Additional File-1).

\section{The conservation of genome structures within serotypes}

A question may arise whether the genome arrangement of D7S-1 is unique and not found in other A. actinomycetemcomitans strains. To address this question we compared the genome arrangements of D7S-1, HK1651 and D11S-1 with those in the contigs of strains D17P-3, ANH9381, and D17P-2 (serotypes a, b and c, respectively). There were significantly fewer intra-contig breakpoints in the comparisons within each serotype than between serotypes $\mathrm{a}$ and $\mathrm{b} / \mathrm{c}$. We identified one intra-contig break point in 267 large contigs of D17P-3 in a pair-wise comparison to D7S-1. Similarly, we found 4 intra-contig breakpoints in 3 of the 102 large contigs of ANH9381 compared to HK1651, and 4 intra-contig breakpoints in 2 of the 62 large contigs of D17P-2 compared to D11S-1. In contrast, we identified 47 breakpoints in 40 contigs of D17P-3 compared to HK1651. The results are consistent with the conservation of the genome arrangement within serotypes, but not between serotypes $\mathrm{a}$ and $\mathrm{b} / \mathrm{c}$.

\section{Distribution patterns of genes and operons in the genomes of $A$. actinomycetemcomitans}

It is possible that the relative gene locations in the genome may be preserved after large-scale genome rearrangements [6]. To address this question in $A$. actinomycetemcomitans, we first identified the Ori and the Ter in the genomes of D7S-1, HK1651 and D11S1 , and analyzed (i) the balance of the replichores, (ii) the gene density in the leading and the lagging strands, and (iii) the positions of the orthologous genes relative to the Ori. We further examined the preservation of the operons in strains of different genome arrangements. 


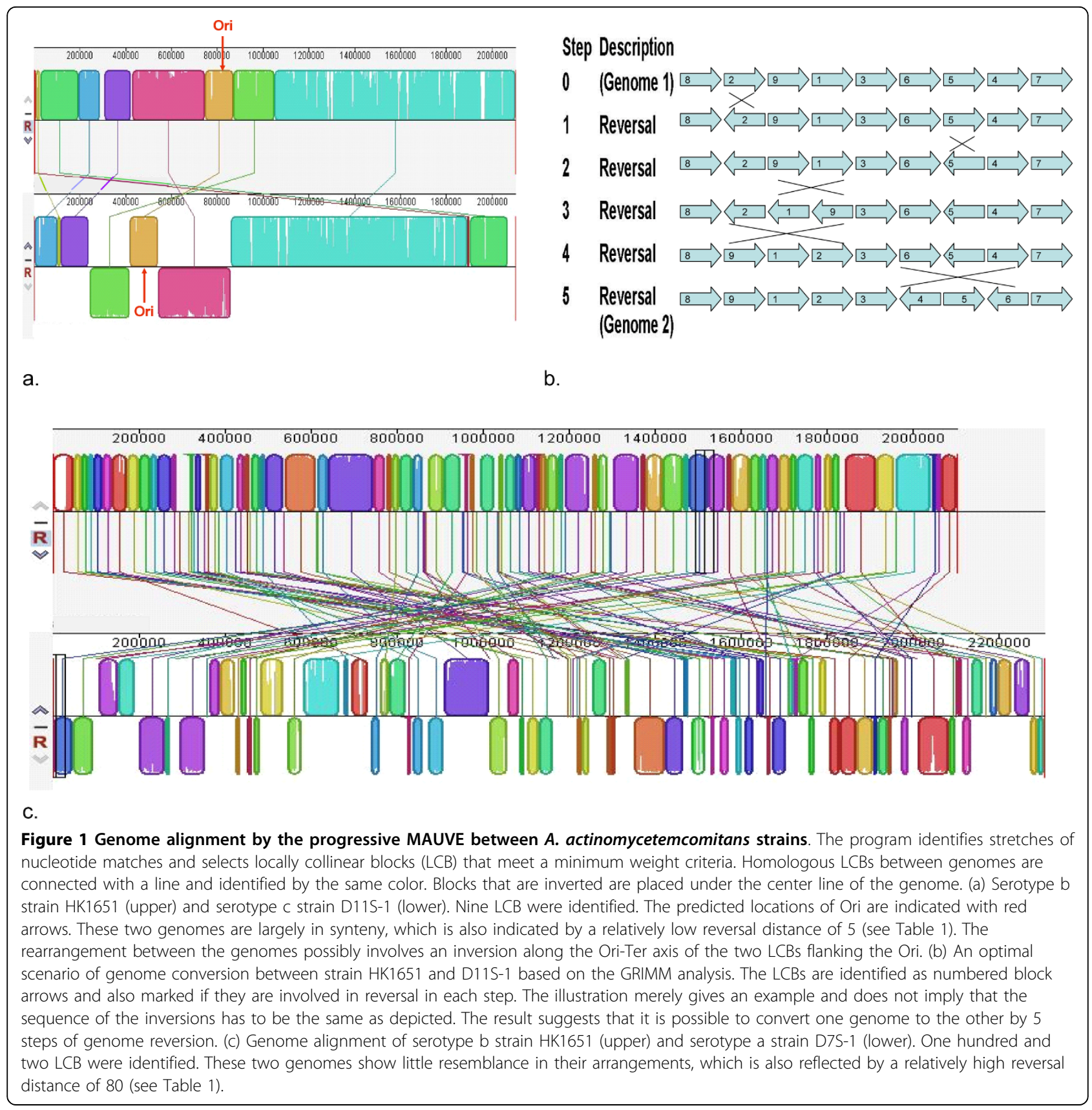

The combined cumulative T-A and C-G skews of the $3^{\text {rd }}$ codons peaked at the nucleotide coordinate $\sim 1,062$ $\mathrm{Kb}$ and declined and changed sign at the nucleotide coordinate $\sim 67 \mathrm{~Kb}$ for D7S-1 (see Additional File 2, top panel). Several peaks of similar heights were identified at coordinates $760 \mathrm{~Kb}, 825 \mathrm{~Kb}$ and $875 \mathrm{~Kb}$ for HK1651, and at $437 \mathrm{~Kb}, 515 \mathrm{~Kb}$ and $550 \mathrm{~Kb}$ for D11S-1 (see Additional File 2, middle and bottom panels). The low- est points of the combined skews changed sign at coordinates $\sim 1,450 \mathrm{~Kb}$ for HK1651 and 1,246 Kb for D11S1. Based on the results we assigned the Ori and the Ter respectively to nucleotide coordinates $1,062,100$ and 67,100 in D7S-1, 825,100 and 1,449,500 in HK1651, and 515,300 and $1,246,100$ in D11S- 1 . The predicted locations of Ori and Ter in HK1651 and D11S-1 were supported by the observation that a large-scale genomic 
Table 1 Genome rearrangement analysis and by progressive MAUVE and GRIMM of bacterial species

\begin{tabular}{lcc}
\hline Bacteria & No. of LCB $^{\mathbf{a}^{\mathbf{2}}}$ & Reversal distance $^{\mathbf{b}^{2}}$ \\
\hline A. actinomycetemcomitans HK1651 vs D7S-1 & 102 & 80 \\
A. actinomycetemcomitans HK1651 vs D11S-1 & 9 & 5 \\
H. somnus 2336 vs 129PT & 51 & 37 \\
H. influenzae Rd KW20 vs 86-028NP & 11 & 6 \\
H. influenzae PittEE vs PittGG & 23 & 17 \\
E. coli 536 vs ATCC8739 & 9 & 5 \\
E. coli O157:H7 EDL933 vs O12:H6 E2348/69 & 9 & 7 \\
E. coli O157:H7 EDL933 vs ATCC8739 & 6 & 3 \\
E. coli CFT073 vs E. coli O157:H7 EDL933 & 21 & 16 \\
N. gonorrhoeae NCCP11945 vs FA1090 & 14 & 10 \\
N. meningitides FAM18 vs MC58 & 16 & 10 \\
N. meningitides Z2491 vs MC58 & 14 & 8 \\
P. gingivalis ATCC33277 vs W83 & 41 & 29 \\
P. aeruginosa PA7 vs PAO1 & 12 & 7 \\
P. aeruginosa LESB58 vs PAO1 & 8 & 5 \\
A. actinomycetemcomitans HK1651 vs A. aphrophilus NJ8700 & 155 & 162 \\
A. actinomycetemcomitans D7S-1 vs A. aphrophilus NJ8700 & 192 & \\
\hline
\end{tabular}

a $\mathrm{LCB}$; locally colinear block identify by the progressive MAUVE

${ }^{\mathrm{b}}$ Reversal distance is determined from the post-analysis of genome rearrangement by the progressive MAUVE using the GRIMM algorithms.

inversion between HK1651 and D11S-1 occurred along the axis of Ori-Ter as commonly observed in other species [8-10].

The imbalance of the replichores was analyzed as described previously (the absolute value of [the length of the replichore-half length of the genome]/half length of the genome) [25]. We found that the imbalance of D7S1 was $13.8 \%$, which may be considered within the normal range of deviations among many bacterial species. In contrast, the imbalance of the replichores in HK1651 and D11S- 1 were $40.1 \%$ and $30.6 \%$, respectively.

The distributions of the predicted genes in the leading and the lagging strands are shown in Table 2 . The gene density is higher in the leading strand than in the lagging strand in the $3 \mathrm{~A}$. actinomycetemcomitans strains of this study. Higher numbers of essential genes were found in the leading strand than in the lagging strand for D7S-1 and HK1651, but D11S-1 showed no strand preference.

Table 2 Distribution of genes in leading and lagging strands in A. actinomycetemcomitans strains

\begin{tabular}{llccc}
\hline Strain & Strand & $\begin{array}{c}\text { Gene } \\
\text { Density }\end{array}$ & $\begin{array}{c}\text { No. of Essential } \\
\text { Gene (\%) }\end{array}$ & $\begin{array}{c}\text { No. of Non-essential } \\
\text { gene (\%) }\end{array}$ \\
\hline D7S-1 & Leading & $46.6 \%$ & $153(59.1)$ & $1171(52.2)$ \\
& Lagging & $42.0 \%$ & $106(40.9)$ & $1074(47.8)$ \\
HK1651 & Leading & $46.2 \%$ & $151(56.8)$ & $1124(51.8)$ \\
& Lagging & $43.9 \%$ & $115(43.2)$ & $1047(48.2)$ \\
D11S-1 & Leading & $45.1 \%$ & $131(50)$ & $1007(51.8)$ \\
& Lagging & $43.5 \%$ & $131(50)$ & $938(48.2)$ \\
\hline
\end{tabular}

The distances of the orthologous genes to the Ori are presented in Figure 2a-f. In Figure 2a the distances of each pair of the orthologous genes in HK1651 and D11S-1 were similar and can be explained by an offset of $\sim 130 \mathrm{~Kb}$ between the genomes (with the exception of 8 genes where the differences in the distances to Ori were $\sim 740 \mathrm{~Kb}$ ). In contrast, the orthologous genes in HK1651 and D7S-1 (Figure 2b) resided in different locations relative to Ori and no specific distribution patterns were found. Similar results were found for subgroups of essential/nonessential and highly expressed/nonhighly expressed genes (Figure $2 \mathrm{c}-\mathrm{f}$ ). Also, there was no discernable tendency for the highly expressed genes to be closer to the Ori than the non-essential genes.

The potential disruptions of operons in the A. actinomycetemcomitans strains were examined. The analysis of operon positions with respect to genomic rearrangement between D7S-1 and HK1651 showed that out of 564 operons in the D7S-1 genome predicted by Database of prOkaryotic OpeRons (DOOR) tool, 558 (98.9\%) were found to be intact in HK1651 and were not affected by the genomic rearrangements between these strains (see Additional File 3 for the list of disrupted operons in D7S-1). Similarly, 505 of 515 (98\%) predicted operons in HK1651 were found to be intact in D7S-1 (see Additional File 3 for the list of disrupted operons in HK1651). For the affected operons, the rearrangement breakpoints occurred between genes of the operons resulting in separations of genes rather than splitting the genes into two fragments. Similar results were obtained using FGENESB (http://linux1.softberry.com/ 


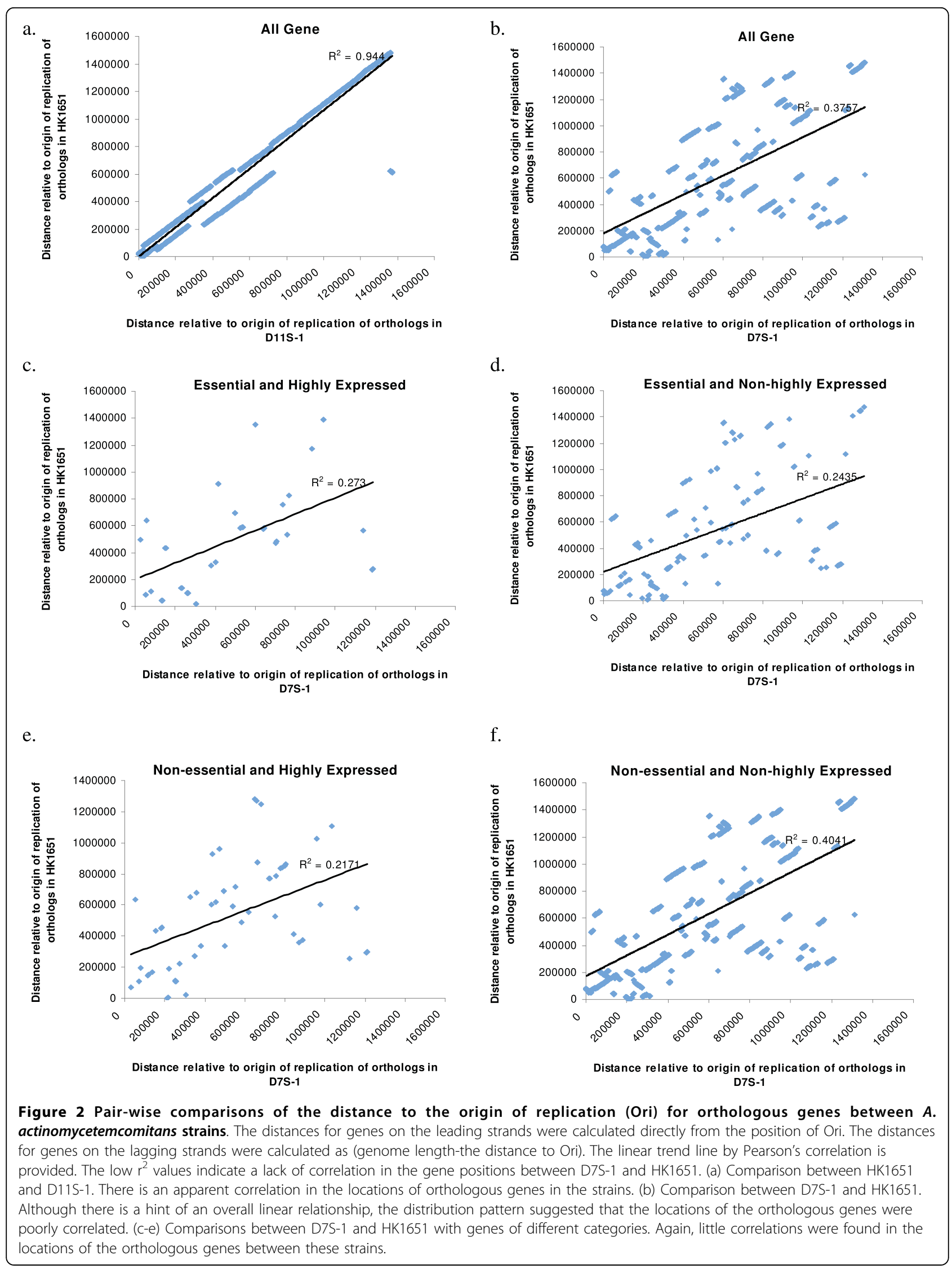


Table 3 Positions and percent of genome occupied by 50 base pair repeat in the genomes of A. actinomycetemcomitans and other bacterial species

\begin{tabular}{lccc}
\hline Genome & $\begin{array}{c}\text { Total number of } \\
\text { position with } \mathbf{5 0} \\
\text { bp } \\
\text { exact matches }\end{array}$ & $\begin{array}{c}\text { Genome } \\
\text { length } \\
\text { (bp) }\end{array}$ & $\begin{array}{c}\text { Percent of } \mathbf{5 0} \text { bp } \\
\text { exact } \\
\text { matches with } \\
\text { respect } \\
\text { to the genome } \\
\text { length }\end{array}$ \\
\hline D7S-1 & 144,825 & $2,308,328$ & $6.27 \%$ \\
HK1651 & 44,531 & $2,105,503$ & $2.11 \%$ \\
D11S-1 & 47,809 & $2,105,764$ & $2.27 \%$ \\
E. coli K12 & 102,642 & $4,639,675$ & $2.21 \%$ \\
P. aeruginosa & 84,973 & $6,264,404$ & $1.36 \%$ \\
PAO1 & & & \\
H. pylori G28 & 41,316 & $1,652,982$ & $2.50 \%$ \\
\hline
\end{tabular}

berry.phtml?topic $=$ fgenesb\&group $=$ programs \&subgroup=gfindb) (data not shown).

\section{Features of genome rearrangement breakpoints}

Genome rearrangements commonly occur via recombination between repeat elements or duplicated genes. We hypothesized that there might be specific features at the inter-LCBs regions and/or the ends of the LCBs that flanked the rearrangement breakpoints. To examine this hypothesis, 50-bp sub-sequences on both strands of each genome were extracted in sliding windows of $1 \mathrm{bp}$, and compared to the entire genome to identify a perfect match in other regions. These 50-base-pair-repeat regions are summarized in Table 3. D7S-1 genome contained a higher number of repeat regions than in HK1651, D11S-1 or other bacteria species analyzed. The percentage of the overlap between the cumulative inter$\mathrm{LCB}$ regions and repeat regions are presented in Table 4. Higher percentages of the genome in D7S-1 were occupied by repeat regions than in the other two strains. Figure 3 illustrates the locations of the repeat elements and the inter-LCB breakpoint regions (relative to HK1651) of D7S-1. The regions between LCBs in D7S-1 were enriched with repeated sequences (See Additional

Table 4 Overlap between repeat and inter-LCB region

\begin{tabular}{lccccc}
\hline Strain & $\begin{array}{c}\text { Total } \\
\text { inter-LCB } \\
\text { length } \\
\text { (bp) }\end{array}$ & $\begin{array}{c}\text { Total } \\
\text { repeat } \\
\text { length } \\
\text { (bp) }\end{array}$ & $\begin{array}{c}\text { Total } \\
\text { overlap } \\
\text { (bp) }\end{array}$ & $\begin{array}{c}\text { Percent of } \\
\text { repeat } \\
\text { overlapping } \\
\text { with inter-LCB }\end{array}$ & $\begin{array}{c}\text { Percent of } \\
\text { inter-LCB } \\
\text { overlapping } \\
\text { with repeat }\end{array}$ \\
\hline D7S-1 $^{a}$ & 251,896 & 159,531 & 105,889 & $66.4 \%$ & $42 \%$ \\
HK1651 $^{\text {b }}$ & 90,658 & 50,603 & 16,729 & $33.1 \%$ & $18.5 \%$ \\
D11S-1 $^{c}$ & 112,883 & 53,208 & 20,973 & $39.4 \%$ & $18.6 \%$ \\
\hline
\end{tabular}

${ }^{a}$ Regions between rearranged conserved blocks (inter-LCB) for D7S-1 are obtained from D7S-1 vs HK1651 MAUVE alignment.

${ }^{b}$ Regions between rearranged conserved blocks (inter-LCB) for HK1651 are obtained from D7S-1 vs HK1651 MAUVE alignment

c Regions between rearranged conserved blocks (inter-LCB) for D11S-1 are obtained from D7S-1 vs D11S-1 MAUVE alignment
File 4 for the locations of the repeat elements and the inter-LCB breakpoint regions of HK1651).

A summary comparison of the frequency and the feature of the repeat elements among strains are shown in Table 5 (see Additional File 5 for sequences of the repeat elements). D7S-1 has more repeat elements than HK1651 or D11S-1. Several of these repeat elements are shared among strains (allowing minor sequence variations). However, many of the repeat elements found in D7S-1 are unique to this strain.

\section{Discussion}

A number of studies have suggested variable virulence among A. actinomycetemcomitans strains [12,15,16, 19,20,26,27]. Most of these studies examined the clinical associations of specific genotypes of $A$. actinomycetemcomitans with periodontal health and disease in crosssectional and prospective studies, but did not provide insight to the molecular basis of such variations. In the present study we provided evidence for variations in the physical arrangement of genes in the genomes of A. actinomycetemcomitans, which may affect the phenotypes or virulence of the strains. Serotypes a-c of A. actinomycetemcomitans were selected for this study because they are frequently identified and may represent up to $80 \%$ of the A. actinomycetemcomitans clinical isolates in human subgingival plaque [13].

The differences in the genome arrangement between strains were visualized with the use of MAUVE and then quantified by GRIMM to calculate the reversal distance. The use of reversal distance in phylogenetic analysis is based on the premise that genomic inversion is the primary type of rearrangement event in bacteria, which was supported by several studies $[8,28]$. Moreover, there is a general correlation between reversal distance and sequence-based phylogenetic analysis [28]. While low reversal distance may be relatively accurate, high reversal distance is likely to underestimate the true phylogenetic distance between genomes.

It is striking and unusual (in comparison to the variations seen in other bacterial species) that the serotype a strain D7S-1 displayed a markedly different genome arrangement relative to HK1651 or D11S-1. The potential sources of errors were examined first. We could rule out large scale sequencing or assembly errors of the contigs based on the results of the PCR analysis of the breakpoints in D7S-1. Moreover, minor sequencing errors would have little or no effect on the genome comparison by MAUVE which examines large homologous blocks between strains. We could also rule out assembly errors because the finished genomes were confirmed with optical mapping [23,24]. The results would not have been affected by the specific locations of the Ori or the Ter in the strains. Additional supporting 


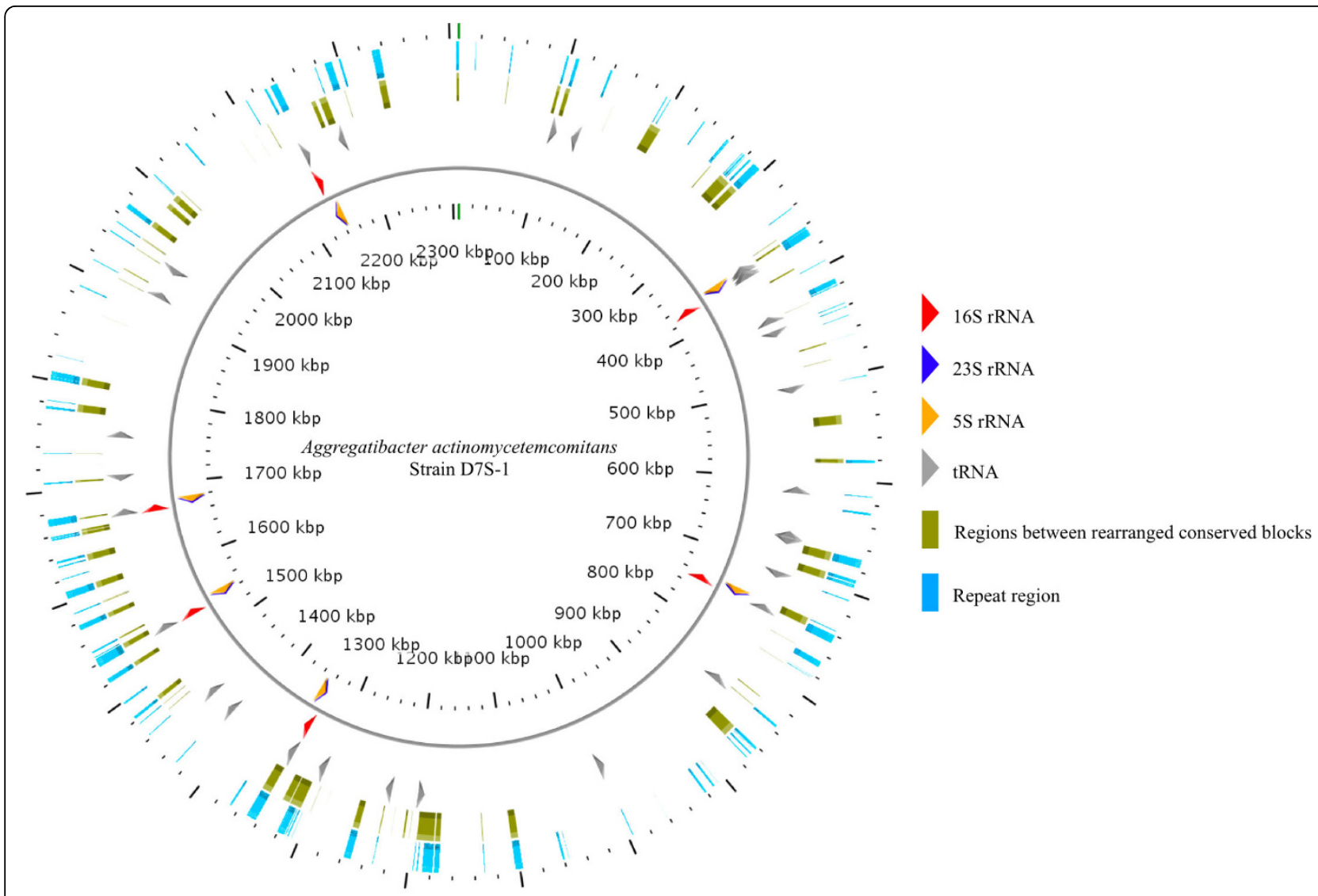

Figure 3 Circular chromosome map of D7S-1 genome. This figure shows that boundaries of the rearranged regions, as suggested by whole genome alignment of D7S-1 and HK1651 genome sequences, are significantly enriched with repeated sequences.

evidence was from the analysis of unscaffolded large contigs of 3 A. actinomycetemcomitans strains. Few intra-contig breakpoints were found between strains of the same serotypes, in contrast to the high numbers of intra-contig breakpoints between strains of serotype a and serotypes $\mathrm{b} / \mathrm{c}$. Therefore we concluded that serotype a strains exhibited markedly different genome arrangements compared to those in serotypes b/c strains, and further suggested that the genome arrangements were conserved within serotypes.

The Ori and the Ter may be identified by various in silico methods based on analyses of DNA asymmetry, distribution of DNA boxes and dnaA gene location [29-32]. The methods based on DNA asymmetry appear to be the most universal and have been used to identify the Ori and Ter of $H$. influenzae (a member of the Pasteurellacea) $[32,33]$. In the absence of experimental determination the locations of the Ori and the Ter of A. actinomycetemcomitans strains identified in this study were in agreement with the available evidence as discussed below.

We noted that in the annotation of HK1651 the origin of replication was not identified but the starting codon of the $d n a A$ gene was assigned the first nucleotide coordinate of the genome. While in some species the location of the $d n a A$ gene coincides with the origin of replication [29], this is not the case in $H$. influenzae and presumably not in A. actinomycetemcomitans either. Eriksen et al [34] showed evidence of intragenomic recombination in JP2 clone of $A$. actinomycetemcomitans via homologous recombination of the 6 rRNA operons and 7 IS150-like repeat elements. It is interesting to note that, with the exception of one case (recombination between IS150-2 and IS150-4), all recombinations occurred along the axis of Ori-Ter predicted in this study. In this study we also found an example of a large-scale genomic inversion between HK1651 and D11S-1 along the axis of Ori-Ter.

Genome rearrangements may occur via homologous recombination of repeat elements in the bacterial genomes [35,36]. A. actinomycetemcomitans genomes contain diverse repeat elements that may mediate genome rearrangement. Within A actinomycetemcomitans genomes some inter-LCB regions were occupied by composites of diverse repeat elements, which may reoccur in other inter-LCB regions but with minor variations 
Table 5 Frequency and features of the repeat elements identified in A. actinomycetemcomitans

\begin{tabular}{|c|c|c|c|c|c|}
\hline Repeat ID & $\begin{array}{l}\text { Estimated } \\
\text { length } \\
\text { (bp) }\end{array}$ & Features & $\begin{array}{c}\text { Frequency } \\
\text { in D7S-1 } \\
\text { Genome }\end{array}$ & $\begin{array}{c}\text { Frequency } \\
\text { in HK1651 } \\
\text { Genome }\end{array}$ & $\begin{array}{c}\text { Frequency } \\
\text { in D11S-1 } \\
\text { Genome }\end{array}$ \\
\hline REPEAT01 & 6505 & rRNA genes operon & 6 & 6 & 6 \\
\hline REPEAT02 & 1269 & IS150 like element & 8 & 7 & 5 \\
\hline REPEAT03 & 717 & IS200 like element & 0 & 4 & 5 \\
\hline REPEAT04 & 139 & Unknown & 1 & 5 & 5 \\
\hline REPEAT05 & 6326 & FHA domain protein & 2 & 0 & 0 \\
\hline REPEAT06 & 4315 & WD-40 repeat, Hypothetical protein and FHA domain protein & 2 & 0 & 0 \\
\hline REPEAT07 & 2156 & Sel1 domain protein repeat-containing protein & 4 & 0 & 0 \\
\hline REPEAT08 & 2008 & Rhs element Vgr protein & 6 & 0 & 0 \\
\hline REPEAT09 & 1523 & Glycoside hydrolase, family 19 gene & 5 & 0 & 0 \\
\hline REPEAT10 & 1254 & FHA domain protein & 2 & 0 & 0 \\
\hline REPEAT11 & 1227 & Tra5 Protein & 6 & 0 & 0 \\
\hline REPEAT12 & 1060 & IS30 like element & 3 & 0 & 0 \\
\hline REPEAT13 & 1225 & Translation elongation factor Tu & 2 & 2 & 2 \\
\hline REPEAT14 & 837 & Putative FHA domain protein & 4 & 0 & 0 \\
\hline REPEAT15 & 690 & IS427 like element & 4 & 0 & 0 \\
\hline REPEAT16 & 472 & Sel1 domain protein repeat-containing protein & 7 & 0 & 0 \\
\hline REPEAT17 & 387 & Hypothetical protein & 10 & 0 & 0 \\
\hline REPEAT18 & 219 & Unknown & 8 & 4 & 2 \\
\hline REPEAT19 & 162 & Hypothetical protein & 7 & 1 & 1 \\
\hline REPEAT20 & 100 & Unknown & 7 & 5 & 5 \\
\hline REPEAT21 & 135 & Autotransporter adhesin Aae & 4 & 3 & 2 \\
\hline
\end{tabular}

(sequence variations, truncation, or absence) of the individual repeat elements. Some of the sequence diversity may be due to sequencing errors. For these reasons we chose to analyze the occurrence of repeat elements with a sliding 50-base window. We noted that A. actinomycetemcomitans D7S-1 has a greater number of repeat elements than HK1651 or D11S-1. The data alone, however, cannot be used to infer the ancestral genome structure of A. actinomycetemcomitans.

Some of the repeat elements are identified in all three A. actinomycetemcomitans strains. The IS150 like elements have been reported previously in the genome of HK1651 [34] and are found in both D7S-1 and D11S-1 in this study. The presence of variable copy numbers of a 135-bp repeat sequence in the autotransporter adhesion gene Aae in different $A$. actinomycetemcomitans strains has been reported previously [37]. There seems to be a distinction in the distribution pattern of the repeat elements in D7S-1 in comparison to that in HK1651/D11S-1. For example, 12 of the repeat elements in D7S-1 are unique and not found in HK1651 or D11S1. Vice versa the REPEAT03 is identified in HK1651 and D11S-1 but not in D7S-1. Also, the copy numbers of some of the repeat elements (REPEAT-04, -19, -20) are identical in HK1651 and D11S-1 and different from the copy numbers of the elements found in D7S-1. Further examination of other A. actinomycetemcomitans strains is needed to determine whether such distribution pattern has any phylogenetic significance.

The results from this study appear to suggest that the genome arrangement of $A$. actinomycetemcomitans strains may be less constrained by cellular processes than in other bacterial species. This could be explained by several factors. The growth rate of $A$. actinomycetemcomitans is comparatively low (doubling time of $\sim 3-4$ hrs in optimum laboratory growth conditions). There might be little or no gene dosage effects and problem of collisions between replication fork and RNA polymerase in slow-growing bacteria, which allow the bacteria to tolerate large-scale genomic rearrangements. The effective population size of some clonal lineages of $A$. actinomycetemcomitans (e.g., serotypes b and c) may be small, which allow these clones to persist in the population. It is also possible that serotypes b and $\mathrm{c}$, as represented by HK1651 and D11S-1, are more recently evolved and have not had sufficient time to allow the mutation pressures to exert their effects. This interpretation is supported by the imbalanced genomes of HK1651 and D11S-1, which could be a consequence of recent changes of their genome arrangements.

While there are significant differences in the genome arrangements in D7S-1 and HK1651 (or D11S-1) they essentially did not affect the operons. However, the locations of orthologous genes were significantly different 
between D7S-1 and HK1651 (or D11S-1). Presumably such differences will affect the phenotypes of the strains. We further noted the replichores were severely unbalanced for strains HK1651 and D11S-1 and less so for strain D7S-1. Evidence has suggested a strong selection for bacteria with a balanced genome [38]. On the contrary, no evidence of natural selection for balanced genomes was found in the analysis of eight Yersinia genomes [25]. We have detected no significant differences in growth rate and biofilm formation under laboratory growth conditions among these 3 A. actinomycetemcomitans strains (unpublished data). The significance of genome arrangement to the phenotypes of A. actinomycetemcomitans remains to be elucidated.

The differences in the genome arrangement or genome content alone may not be sufficient to determine whether some $A$. actinomycetemcomitans strains should be designated a subspecies or even a new species. There appear to be no universally accepted concept and definition of bacterial species. With the advancement of bacterial genomics various approaches for species definition have been proposed that combine the analyses of the $16 \mathrm{~S}$ rRNA gene sequence identity, DNA-DNA hybridization, percentage of the shared genes in the genome, the average nucleotide identity (ANI) of the shared genes and ecological factors [39,40]. Pair-wise comparison of the 16S rRNA gene sequences in strains D7S-1, HK1651 and D11S-1 showed $>97.6 \%$ nucleotide identity, which is within the accepted working definition of all three being from the same species. We are analyzing the genome contents of the sequenced serotypes a-f strains to further address this question.

In addition to the potential biological impact of the observed large-scale genomic rearrangement between serotype a strains and serotype b/c strains, there are also implications of the rearrangement on a practical research level. In the early stages of our assembly and finishing of the D7S-1 genome we had hoped to use the HK1651 genome as a guide to assist in the ordering of contigs. However, the level of genomic rearrangement between these two strains negated the utility of HK1651 as a reference genome for the structure of D7S-1. Also, the variation in genome structure means that PCR products predicted in one strain may cross a breakpoint in another strain and hence will not be amplified in the other strain. It is unclear how frequently similar problems will arise in the sequencing and analysis of other bacterial genomes, but it is worth noting that at least in A. actinomycetemcomitans massive variation in genome structure between strains can lead to confusion in some kinds of analyses.

\section{Conclusions}

A. actinomycetemcomitans serotype a strains display markedly different physical arrangement of genes in comparison to serotype b or c strains. This likely indicates significant differences in the evolutionary history between serotype a strains and serotype b/c strains. The results have provided significant insight to the evolutionary divergence of $A$. actinomycetemcomitans of different serotypes. Also, the serotype-specific genome arrangement patterns have practical application for future genome sequencing of $A$. actinomycetemcomitans.

\section{Methods \\ Bacterial strains}

Serotype a strains D7S-1, D17P-3, and serotype c strains D11S-1 and D17P-2 were cultivated from subgingival plaque of patients with aggressive periodontitis [13,41]. Serotype b strain ANH9381 was recovered from a subgingival plaque sample of a periodontally non-diseased subject. Species identity and serotypes were examined by a $16 \mathrm{~S}$ rRNA-based PCR analysis and a serotype analysis by a PCR-method as described previously [42].

\section{Genome sequences}

The genome sequencing of D7S-1 (one contig; genome size 2,308,328 bp) and D11S-1 (circularized; genome size $2,105,764 \mathrm{bp}$ ) were completed as described previously $[23,24]$. The genome information of the sequenced strain HK1651 (genome size 2,105,503 bp) is accessible from University of Oklahoma (http://www.genome.ou.edu/act. html) and Oralgen (http://www.oralgen.lanl.gov/oralgen/ bacteria/aact/). Contigs generated by 454 sequencing of strains D17P-3 (25× coverage), ANH9381 (16X) and D17P-2 (28X) were also included in the analyses. Additional bacterial genome sequences were downloaded from Genbank for analyses that included Haemophilus somnus strains 2336 and 129PT, Haemophilus influenzae strains RdKW20, 86-028NP, PittEE and PittGG, Escherichia coli strains 536, ATCC8739, O157:H7 EDL933, O12: H6 E2348/69, CFT073 and K12, Neisseria gonorrhoeae NCCP11945 and FA1090, Neisseria meningitidis FAM18, MC58, Z2491, Porphyromonas gingivalis ATCC33277 and W83, Pseudomonas aeruginosa PA7, PAO1, LESB58, Aggregatibacter aphrophilus NJ8700, and Helicobacter pylori G28.

\section{Annotation and comparison of A. actinomycetemcomitans genomes}

A gene prediction and annotation pipeline was put together to process the genome sequence data obtained from the Roche/454 platform and strain HK1651. The gene identification and functional annotation mostly followed the protocol developed by The Institute for Genomic Research (J. Craig Venter Institute). Specifically, protein-coding genes were identified using Glimmer3 software [43] with our custom modification of the predicted results. Similarly, rRNA and tRNA coding 
genes were identified by using Exonerate [44] and tRNAscanSE [45] softwares, respectively. The predicted genes were annotated by first comparing them to the HK1651 annotation using the NCBI BLAST software [46]. Genes that are annotated as hypothetical as well as those that are not present in strain HK1651 were then blasted against Genbank non-redundant protein sequence database. The description of the best BLAST hit is then used as annotation for that gene. The gene orthologs among the $3 \mathrm{~A}$. actinomycetemcomitans strains were identified based on all against all BLAST search. The genes that fulfill the following criteria are included as core genes with: (i) sequence similarity of at least $85 \%$ (ii) length difference of not more than $5 \%$. Pseudogenes and genes with frameshift mutations were excluded from the analysis.

\section{Analysis of genomic rearrangement by MAUVE}

The Progressive Mauve algorithm was used to create the whole genome alignment between different strains of A. actinomycetemcomitans [47]. GRIMM genome rearrangement algorithms were used to obtain the reversal distance between genomes [48].

\section{PCR analysis of genome breakpoints}

The genome breakpoints in D7S-1 were analyzed by PCR. Briefly, 20-mer oligonucleotides were designed with the program Primer 3 [49]. A standard PCR protocol was employed under the following conditions: $5 \mathrm{~min}$ at $94^{\circ} \mathrm{C}$ for denaturation followed by 30 cycles of $94^{\circ} \mathrm{C}$ for $30 \mathrm{sec}$, an annealing step at $60^{\circ} \mathrm{C}$ for $1 \mathrm{~min}$, an extension step at $72^{\circ} \mathrm{C}$ for $2 \mathrm{~min}$ and then a final extension of $10 \mathrm{~min}$ at $72^{\circ} \mathrm{C}$ [50]. PCR amplicons were analyzed by $1 \%$ agarose gel electrophoresis. For sequencing, the PCR products were purified by GIAquick PCR purification kit and GIAquick Gel Extraction kit (Qiagen, Valencia, CA) and submitted for sequencing at the USC School of Medicine Microchemical Core Facility.

\section{Assignment of gene categories in}

\section{A. actinomycetemcomitans}

All open reading frames (ORFs) identified in A. actinomycetemcomitans strains D7S-1, HK1651 and D11S-1 were categorized as essential or non-essential genes. The essential genes were identified based on the Profiling of Escherichia coli chromosome (PEC) database [51]. Specifically, A. actinomycetemcomitans ORFs that are homologous to the essential genes in PEC were considered as essential (blastp with E-value $<=1 \mathrm{e}-6$ ). The remaining ORFs in A. actinomycetemcomitans were considered non-essential by default. ORFs were also classified into highly expressed and non-highly expressed genes based on the codon adaptation index (CAI) calculated using CAIJava tool [52]. ORFs within the top 5 percent highest CAI score are assigned as highly expressed and the remaining ORFs are considered as non-highly expressed.

\section{Analysis of gene density and gene positions}

Combined G-C and T-A skews were first used to predict the locations of the Ori and the Ter with Oriloc [31]. Each genome was then divided into two replichores. The distribution of genes of different categories (essential/non-essential, highly expressed/non-highly expressed) and their densities in the leading and the lagging strands were compared between strains. The Pearson's correlation coefficient of the distance to the Ori for orthologous genes between strains was calculated.

\section{Analysis of preservation of operons among strains}

The operons in A. actinomycetemcomitans were identified by using the Database of prOkaryotic OpeRons (DOOR) tool $[53,54]$ and by FGENESB, a suite of bacterial operon and gene prediction programs [55]. In brief, the DOOR tool predicts bacterial gene operons using a classifier algorithm on features such as intergenic distance, neighborhood conservation, phylogenetic distance, information from short DNA motifs, similarity score between GO terms of gene pairs and length ratio between a pair of genes. The FGENESB tool predicts gene operon based on distances between open reading frames and frequencies of different genes neighboring each other in known bacterial genomes, as well as on promoter and terminator predictions. The positions of predicted operons in one strain were examined in the other strains to identify those that are affected by the genome rearrangement.

\section{Identification of regions with repeat sequences}

A 50-base pair window (each window is a sliding of 1 base pair along the genome sequence) is compared to the entire genome to identify perfect matches (or a perfect match) on any other regions of the genome.

\section{Additional material}

Additional file 1: PDF PCR analysis of genome breakpoints of strain D7S-1. The table provides the PCR primer sequences, the nucleotide coordinates of the PCR target sites in the genome of D7S-1, and the PCR results

Additional file 2: PDF Predicted Ori and Ter positions in $\boldsymbol{A}$. actinomycetemcomitans. The figures show the T-A and C-G skew analysis of the genomes of strains D7S-1, HK1651 and D11S-1.

Additional file 3: PDF Predicted operons in $A$.

actinomycetemcomitans. Two tables show the predicted operons in D7S-1 that are affected by the genomic rearrangement relative to HK1651 and vice versa.

Additional file 4: PDF Locations of repeat elements and inter-LCB regions in strain HK1651. The figure shows the location of the repeat elements in the genome of HK1651 
Additional file 5: PDF Repeat elements in A. actinomycetemcomitans The FASTA sequences of the repeat elements in $A$. actinomycetemcomitans strains D7S-1, HK1651 and D11S-1

\section{Acknowledgements}

This study was supported by NIDCR grant R01 DE12212. The authors wish to thank Aaron Darling for helpful discussions.

The Whole Genome Shotgun projects (strains D17P-3, ANH9381, and D17P2) have been deposited at DDBJ/EMBL/GenBank under the accession ADOA00000000 (A. actinomycetemcomitans D17P-3), ADOC00000000 (A. actinomycetemcomitans ANH9381), and ADOB00000000 (A. actinomycetemcomitans D17P-2).

\section{Author details}

'Department of Microbiology, University of Washington, Seattle, WA, USA. ${ }^{2}$ Division of Periodontology, Diagnostic Sciences and Dental Hygiene, Herman Ostrow School of Dentistry of the University of Southern California, Los Angeles, CA, USA.

\section{Authors' contributions}

WK participated in genome sequencing, gene annotations, identification of genome organization features, performed analysis of gene positions between strains, and identified and characterized repeated sequences of the genomes. RB led the efforts in genome sequencing, established and implemented the protocols for gene annotation and display, and led efforts in comparative genomic analysis between strains. CC conceived of the study and coordinated efforts of the project, performed genome arrangement analyses, and helped draft and finalized the manuscript. All authors contributed equally in data analysis and interpretation, and have read and approved the final manuscript.

Received: 10 May 2010 Accepted: 8 September 2010 Published: 8 September 2010

\section{References}

1. Hacker J, Carniel E: Ecological fitness, genomic islands and bacterial pathogenicity. A Darwinian view of the evolution of microbes. EMBO Rep 2001, 2:376-381.

2. Hacker J, Blum-Oehler G, Muhldorfer I, Tschape H: Pathogenicity islands of virulent bacteria: structure, function and impact on microbial evolution. Mol Microbiol 1997, 23:1089-1097.

3. Hacker J, Hentschel U, Dobrindt U: Prokaryotic chromosomes and disease. Science 2003, 301:790-793.

4. Rocha EP: Inference and analysis of the relative stability of bacterial chromosomes. Mol Biol Evol 2006, 23:513-522.

5. Hill CW, Harnish BW: Inversions between ribosomal RNA genes of Escherichia coli. Proc Natl Acad Sci USA 1981, 78:7069-7072.

6. Rocha EP: The organization of the bacterial genome. Annu Rev Genet 2008, 42:211-233.

7. Rocha EP: Order and disorder in bacterial genomes. Curr Opin Microbiol 2004, 7:519-527.

8. Hughes D: Evaluating genome dynamics: the constraints on rearrangements within bacterial genomes. Genome Biol 2000, 1: REVIEWSO006.

9. Tillier ER, Collins RA: Genome rearrangement by replication-directed translocation. Nat Genet 2000, 26:195-197.

10. Eisen JA, Heidelberg JF, White O, Salzberg SL: Evidence for symmetric chromosomal inversions around the replication origin in bacteria. Genome Biol 2000, 1:RESEARCH0011.

11. Tanner A, Maiden MFJ, Paster BJ, Dewhirst FE: The impact of $16 \mathrm{~S}$ ribosomal RNA-based phylogeny on the taxonomy of oral bacteria. Periodontology 2000 1994, 5:26-51.

12. Rylev $\mathrm{M}$, Kilian $\mathrm{M}$ : Prevalence and distribution of principal periodontal pathogens worldwide. J Clin Periodontol 2008, 35:346-361.

13. Chen C, Wang T, Chen W: Occurrence of Aggregatibacter actinomycetemcomitans serotypes in subgingival plaque from United States subjects. Molecular Oral Microbiology 2010, 25:207-214.
14. Asikainen S, Chen C, Slots J: Actinobacillus actinomycetemcomitans genotypes in relation to serotypes and periodontal status. Oral Microbiol Immunol 1995, 10:65-68.

15. Asikainen S, Lai CH, Alaluusua S, Slots J: Distribution of Actinobacillus actinomycetemcomitans serotypes in periodontal health and disease. Oral Microbiol Immunol 1991, 6:115-118.

16. DiRienzo JM, Slots J, Sixou M, Sol MA, Harmon R, McKay TL: Specific genetic variants of Actinobacillus actinomycetemcomitans correlate with disease and health in a regional population of families with localized juvenile periodontitis. Infect Immun 1994, 62:3058-3065.

17. DiRienzo JM, McKay TL: Identification and characterization of genetic cluster groups of Actinobacillus actinomycetemcomitans isolated from the human oral cavity. J Clin Microbiol 1994, 32:75-81.

18. Haubek D, Poulsen K, Asikainen S, Kilian M: Evidence for absence in Northern Europe of especially virulent clonal types of Actinobacillus actinomycetemcomitans. J Clin Microbiol 1995, 33:395-401.

19. Haubek D, Poulsen K, Westergaard J, Dahlen G, Kilian M: Highly toxic clone of Actinobacillus actinomycetemcomitans in geographically widespread cases of juvenile periodontitis in adolescents of African origin. $J$ Clinic Microbiol 1996, 34:1576-1578.

20. Haubek D, DiRienzo JM, Tinoco EM, Westergaard J, Lopez NJ, Chung CP, Poulsen K, Kilian M: Racial tropism of a highly toxic clone of Actinobacillus actinomycetemcomitans associated with juvenile periodontitis. J Clin Microbiol 1997, 35:3037-3042.

21. Kaplan JB, Schreiner HC, Furgang D, Fine DH: Population structure and genetic diversity of Actinobacillus actinomycetemcomitans strains isolated from localized juvenile periodontitis patients. J Clin Microbiol 2002, 40:1181-1187.

22. Socransky SS, Haffajee AD, Cugini MA, Smith C, Kent RL Jr: Microbial complexes in subgingival plaque. J Clin Periodontol 1998, 25:134-144

23. Chen C, Kittichotirat W, Si Y, Bumgarner R: Genome sequence of Aggregatibacter actinomycetemcomitans serotype c strain D11S-1. J Bacteriol 2009, 191:7378-7379.

24. Chen C, Kittichotirat W, Chen W, Downey JS, Si Y, Bumgarner R: Genome sequence of a naturally competent Aggregatibacter actinomycetemcomitans serotype a strain D7S-1. J Bacteriol 2010.

25. Darling AE, Miklos I, Ragan MA: Dynamics of genome rearrangement in bacterial populations. PLoS Genet 2008, 4:e1000128.

26. Haubek D, Ennibi OK, Poulsen K, Vaeth M, Poulsen S, Kilian M: Risk of aggressive periodontitis in adolescent carriers of the JP2 clone of Aggregatibacter (Actinobacillus) actinomycetemcomitans in Morocco: a prospective longitudinal cohort study. Lancet 2008, 371:237-242.

27. Asikainen S, Chen C, Saarela M, Saxen L, Slots J: Clonal specificity of Actinobacillus actinomycetemcomitans in destructive periodontal disease. Clin Infect Dis 1997, 25(Suppl 2):S227-229.

28. Belda E, Moya A, Silva FJ: Genome Rearrangement Distances and Gene Order Phylogeny in \{gamma\}-Proteobacteria. Mol Biol Evol 2005, 22:1456-1467.

29. Mackiewicz P, Zakrzewska-Czerwinska J, Zawilak A, Dudek MR, Cebrat S: Where does bacterial replication start? Rules for predicting the oriC region. Nucleic Acids Res 2004, 32:3781-3791.

30. Worning P, Jensen $L$, Hallin PF, Staerfeldt HH, Ussery DW: Origin of replication in circular prokaryotic chromosomes. Environ Microbiol 2006, 8:353-361

31. Frank AC, Lobry JR: Oriloc: prediction of replication boundaries in unannotated bacterial chromosomes. Bioinformatics 2000, 16:560-561.

32. Lobry JR: Asymmetric substitution patterns in the two DNA strands of bacteria. Mol Biol Evol 1996, 13:660-665.

33. Fleischmann RD, Adams MD, White O, Clayton RA, Kirkness EF, Kerlavage AR, Bult CJ, Tomb JF, Dougherty BA, Merrick JM, et al: Wholegenome random sequencing and assembly of Haemophilus influenzae Rd. Science 1995, 269:496-512.

34. Eriksen KT, Haubek D, Poulsen K: Intragenomic recombination in the highly leukotoxic JP2 clone of Actinobacillus actinomycetemcomitans. Microbiology 2005, 151:3371-3379.

35. Aras RA, Kang J, Tschumi Al, Harasaki Y, Blaser MJ: Extensive repetitive DNA facilitates prokaryotic genome plasticity. Proc Natl Acad Sci USA 2003, 100:13579-13584.

36. Mira A, Klasson L, Andersson SG: Microbial genome evolution: sources of variability. Curr Opin Microbiol 2002, 5:506-512. 
37. Rose JE, Meyer DH, Fives-Taylor PM: Aae, an autotransporter involved in adhesion of Actinobacillus actinomycetemcomitans to epithelial cells. Infect Immun 2003, 71:2384-2393.

38. Liu GR, Liu WQ, Johnston RN, Sanderson KE, Li SX, Liu SL: Genome plasticity and ori-ter rebalancing in Salmonella typhi. Mol Biol Evol 2006, 23:365-371.

39. Konstantinidis KT, Ramette A, Tiedje JM: The bacterial species definition in the genomic era. Philos Trans R Soc Lond B Biol Sci 2006, 361:1929-1940.

40. Richter M, Rossello-Mora R: Shifting the genomic gold standard for the prokaryotic species definition. Proc Natl Acad Sci USA 2009, 106:19126-19131.

41. Wang Y, Goodman SD, Redfield RJ, Chen C: Natural transformation and DNA uptake signal sequences in Actinobacillus actinomycetemcomitans. J Bacteriol 2002, 184:3442-3449.

42. Fujise O, Lakio L, Wang Y, Asikainen S, Chen C: Clonal distribution of natural competence in Actinobacillus actinomycetemcomitans. Oral Microbiol Immunol 2004, 19:340-342.

43. Delcher $A L$, Bratke $K A$, Powers EC, Salzberg SL: Identifying bacterial genes and endosymbiont DNA with Glimmer. Bioinformatics 2007, 23:673-679.

44. Slater GS, Birney E: Automated generation of heuristics for biological sequence comparison. BMC Bioinformatics 2005, 6:31.

45. Lowe TM, Eddy SR: tRNAscan-SE: a program for improved detection of transfer RNA genes in genomic sequence. Nucleic Acids Res 1997 25:955-964.

46. Altschul SF, Gish W, Miller W, Myers EW, Lipman DJ: Basic local alignment search tool. J Mol Biol 1990, 215:403-410.

47. Darling AC, Mau B, Blattner FR, Perna NT: Mauve: multiple alignment of conserved genomic sequence with rearrangements. Genome Res 2004, 14:1394-1403.

48. Tesler G: GRIMM: genome rearrangements web server. Bioinformatics 2002, 18:492-493.

49. Rozen S, Skaletsky HJ: Primer3 on the www for general users and for biologist programmers. In Bioinformatics Methods and Protocols: Methods in Molecular Biology. Edited by: Krawetz S, Misener S. Totowa, NJ: Humana Press; 2000:365-386.

50. Chen $W$, Wang $Y$, Chen C: Identification of a genomic island of Actinobacillus actinomycetemcomitans. J Periodontol 2005, 76:2052-2060

51. Profiling of Escherichia coli chromosome (PEC) database. [http://www. shigen.nig.ac.jp/ecoli/pec/index.jsp].

52. Carbone A, Zinovyev A, Kepes F: Codon adaptation index as a measure of dominating codon bias. Bioinformatics 2003, 19:2005-2015.

53. Database of prOkaryotic OpeRons (DOOR) tool. [http://csbl1.bmb.uga.edu/ OperonDB/operon_prediction.php].

54. Mao F, Dam P, Chou J, Olman V, Xu Y: DOOR: a database for prokaryotic operons. Nucleic Acids Res 2009, 37:D459-463.

55. FGENESB. [http://linux1.softberry.com/berry.phtml? topic=fgenesb\&group=programs\&subgroup=gfindb].

doi:10.1186/1471-2164-11-489

Cite this article as: Kittichotirat et al:: Markedly different genome arrangements between serotype a strains and serotypes b or c strains of Aggregatibacter actinomycetemcomitans. BMC Genomics 2010 11:489.

\section{Submit your next manuscript to BioMed Central and take full advantage of:}

- Convenient online submission

- Thorough peer review

- No space constraints or color figure charges

- Immediate publication on acceptance

- Inclusion in PubMed, CAS, Scopus and Google Scholar

- Research which is freely available for redistribution

Submit your manuscript at www biomedcentral com/submit 